\title{
Variants of the Course of Proliferative Processes in Patients with Diabetic Retinopathy and Recommendations for its Treatment
}

\author{
Dmitry Lipatov*, Anatoly Kuzmin, Anna Tolkachevf and Timofey Chistyakov \\ National Endocrinology Research Center, Moscow, Russia
}

\section{Article Info}

\section{*Corresponding author:}

Dmitry Lipatov

Head of Department Ophthalmology

National Endocrinology Research Center, Moscow, Russia

E-mail: glas1966@rambler.ru

\section{Received: April 4, 2016}

Accepted: June 9, 2016

Published: June 23, 2016

Citation: Lipatov D, Kuzmin A, Tolkachevf A, Chistyakov T. Variants of the Course of Proliferative Processes in Patients with Diabetic Retinopathy and Recommendations for its Treatment. Madridge J Ophthalmol. 2016; 1(1): 7-9.

doi: 10.18689/mjop-1000103

Copyright: (c) 2016 The Author(s). This work is licensed under a Creative Commons Attribution 4.0 International License, which permits unrestricted use, distribution, and reproduction in any medium, provided the original work is properly cited.

Published by Madridge Publishers

\section{Summary}

Relevance: The pandemic spread of diabetes across the planet has led to the increase in the number and changing the structure of the eye in this disease. The emergence of new modern methods of diagnosis and treatment of diabetic retinopathy, unfortunately, has not led to fewer eye complications in patients with diabetes mellitus. This is due to the fact that the proliferative process in diabetic retinopathy has become more variable - growth of proliferative tissue can go almost all structures of the eyeball. This complicates the timely diagnosis, delay the starting time of treatment and, ultimately, worsens the prognosis of the disease.

Purpose: Generalization of data on variants of the course of the proliferative process in diabetic retinopathy.

Material and Methods: The study centered on patients with diabetic retinopathy of various regions of the Russian Federation, held examination and treatment in the Department of Ophthalmology of National Endocrinology Research center (Moscow, Russia) in 2011-2015 inclusive.

Conclusion: The Extension of our knowledge about types of the course of the proliferative process in diabetic retinopathy allows us to diagnose pathological changes in the eye and timely treatment. All of this can contribute to a more optimistic prognosis of the disease.

Keywords: Diabetes mellitus; Diabetic retinopathy; Neovascular glaucoma; Proliferative process.

Abbreviations: AChA: Anterior Chamber Angle; AHM: Anterior Hyaloid Membrane; DM: Diabetes Mellitus; DME: Diabetic Macular Edema; DR: Diabetic Retinopathy; DRS: Diabetic Retinopathy Study, 1972-1976; ETDRS: Early Treatment Diabetic Retinopathy Study, 1980-1990; IOP: Intraocular Pressure; LCR: Laser Coagulation of the Retina; NG: Neovascular Glaucoma; PHM: Posterior Hyaloid Membrane.

\section{Relevance}

According to IDF [1] by the end of 2015, there were more than 400 million patients with diabetes mellitus (DM). In Russia show similar trends. According to the official register in the Russian Federation on 01.01.2016 there more than 4 million patients with DM. However, the actual number of these patients is $2-2.5$ times registered and is approaching $6-7 \%$ of the population. Almost one fifth of these patients (19.85\%) has problems with vision. Most of them $(70-75 \%)$ are patients with varying degrees actually diabetic retinopathy (DR), lower (20-25\%) - with complicated diabetic cataract, secondary neovascular glaucoma (NG) chronic diseases of the eyelid margins, transient visual impairments, and other problems [4]. 
The development of proliferative changes in the posterior segment of the eye (so-called "rear" proliferation) is characterized by the growth of abnormal, morphologically defective vessels of the posterior hyaloid membrane (PHM) of the vitreous with subsequent development of hemorrhage, fibrosis and retinal detachment. Neovascularization of the anterior segment of the eye (so-called "front" proliferation) leads to a change in the structure of anterior chamber angle (AChA), the violation of the outflow of intraocular fluid significant increase in intraocular pressure (IOP) and ultimately to the development of secondary neovascular glaucoma, which is one of the most severe forms of glaucoma and belongs to one of the terminal stages of proliferative $D R$ in DM patients. According to our data, it is diagnosed in $0.3 \%$ of patients referred for consultation with an ophthalmologist about the eye complications of diabetes mellitus [3].

Functionally defective newly formed vessels are characterized by rapid growth, massive extravasatiore of plasma and increased fragility, which leads to hemorrhage. In the development of blindness is essential doring from newly formed vessels of the protein fractions of blood plasma, which start a process of scarring in the tissues of the iris, retina and vitreous, which ultimately may be causing traction syndrome. Sometimes proliferation occurs atypical - abnormal blood vessels grow on the compacted anterior hyaloid membrane (AHM) of the vitreous body (so-called "average" proliferation). In some cases, the process may be of a composite nature (a combination of certain types of pathologic neovascularization), others to be independent and local symptom manifestations of proliferative retinopathy.

In this regard, the aim of this work was the synthesis of their own data and observations, variants of the course of the proliferative process in diabetic retinopathy.

\section{Material and Methods}

The patients from different regions of Russia with DM type 1 and 2 were investigated seeking help regarding problems with vision in Department of Ophthalmology of National Endocrinology Research center (Moscow, Russia) for the period 2011-2015 inclusive. During this time we have consulted more than 32.000 , of which about 27.000 were patients with DM (85\%) of both types. DM type 2 was $92.0 \%$, the ratio of women to men is $66 \%$ and $34 \%$, respectively, with an average age of about 68 years.

\section{Discussion}

"Classical" development of proliferative process in the eyeball in patients with DM is in the posterior segment of the eye and conditionally can be attributed to the "rear" of proliferation. These patients were identified about 2.000 people, which accounted for $6.8 \%$ of all patients with DM [4].

Proliferative DR is characterized by the appearance of newly formed vessels not only across the field of retina, and optic nerve head with their intense penetration from the vitreous body. Numerous studies have shown that newly formed vessels grow mainly along posterior (retinal) surface of the posterior hyaloid membrane (PHM). In the presence of defects in PHM, proliferative tissue delaminates, or it grows on the front surface of the membrane. Growth of newly formed vessels outside of PHM was not detected. The detachment of the PHM newly formed vessels ophthalmoscopically simulates growth into the vitreous body (VB). In the absence of detachment of the PHM, the newly formed vessels grow in the plane of the retina between the internal limiting membrane and the PHM, being located on the surface of the retina. In the process of development of proliferative DR, PHM and the newly formed epiretinal tissue is almost indistinguishable among themselves. It is proved that a complete detachment of the PHM vitreous body prevents the development of proliferative DR. Therefore the main task of vitreoretinal surgery - the complete removal of PHM as a substrate for the growth of newly formed vessels.

Treatment of proliferative process in any part of the eyeball, and other late complications of DM, based on optimal compensation for the patient along with the normalization of fat, protein and water-salt metabolism. One of the effective methods of treatment of the rear of proliferation has been and remains the laser coagulation of the retina (LCR). Its timely in adequate amounts helps to stabilize the process in $70-75 \%$ of cases even in the proliferative stage of the disease.

If the pathological process is limited to individual sites, conducted focal LCR within the affected areas. The panretinal LCR appeid with extensive changed and a proliferation. Treatment is carried out in several stages (sessions) under the control of fluorescent angiography (FAG). LCR purpose - the termination of defective functioning of newly formed vessels, which pose the main threat to the development of severe complications such as hemophthalmos, retinal detachment, robes of the iris and secondary glaucoma. Significant bleeding, the presence of a dense proliferates in the vitreous body, opacity of the lens make it difficult, and sometimes make it impossible for $L C R$.

With the growth of technical equipment of ophthalmology in general and surgery in particular, is actively developing another effective method of treatment in the proliferative stage of DR - vitrectomy - removal of pathologically disease vitreous body. It is indicated in massive, long-term nonabsorbable case of hemophthalmias, traction syndrome, retinal detachment. Hemorrhage in the only seeing eye and bilateral hemophthalmus is also considered an indication for urgent re. For the decision of a question on tactics of treatment, and the possibility of the choice of anesthesia necessary coordinated work of the ophthalmologist, endocrinologist and anesthesiologist.

Currently obtained a good effect in the treatment of diabetic macular edema (DME), which occurs in any stage of $D R$, the use of a new group of drugs - inhibitors of the angiogenic effect (antagonists of VEGF-factor). The obtained results are encouraging for the possibility of holding a future conservative or combined (with surgery), treatment DR. 
Today, it is difficult to imagine that Rubeus iris or neovascularization literally three decades ago was considered a rare pathology of the anterior eye. This disease is usually combined with vascular diseases of the retina, and its relation to secondary neovascular glaucoma (NG), it was unclear. Hemorrhagic glaucoma observed in occlusion of the central retinal vein, was considered different from robosnail glaucoma observed in patients with diabetes. Due to the growth of vascular pathology of the body, increasing longevity of the population in economically developed countries, especially in diabetic patients, and to improve the quality of diagnosis - neovascularization of the iris has come to light more often. With a certain degree of conditionality can be called "front" proliferation.

In the initial stage of this kind of proliferative process at biomicroscopy revealed dilated branches of capillaries and fine, randomly oriented vessels on the surface of the iris on pupillary edge. As shown by histopathological studies, the growth of newly formed vessels intrastromal begins, and then extends to the surface of the iris. Neovascularization in most cases (80\%) first appears on pupillary edge of the iris. The formation of new blood vessels extends towards the AChA. Although the clinical picture of rubiosis the same as in the case of diabetic eye disease, and in the case of occlusion of the $V A B$, the study revealed more pronounced and widespread rubeosis in the eyes of DM patients. For newly formed vessels are characterized by the presence of thin fenestrated walls, variability of size and tortuous course. Spreading to the periphery, they cut across ciliary body, scleral spur, unlike their own vessels, which are located posterior to the scleral spur.

Therapy NG can be divided into conservative (drug) treatment of radiation exposure and surgery. Despite the fact that currently there is a wide range of local drugs of the hypotensive action, their efficacy in the treatment of NG is low. The high efficiency of combined use of drugs and the disappointing results from drug monotherapy [3]. To ensure pain NG used contact with metered indentation of the sclera and non-contact trans-scleral diod-laser cyclocoagulation, in which, as a rule, achieved relief of pain, and the hypotensive effect is not always predictable. To date, the accepted method of locking the ischemic zones and stabilization of the eyes with DR in General and NG in particular remains panretinal $L C R$, clinical effectiveness has been confirmed by large scale prospective studies in the DRS (Diabetic Retinopathy Study, 1972-1976) and ETDRS (Early Treatment Diabetic Retinopathy Study, 1980-1990).

The use of classic glaucoma surgery (sinus-trabeculaectomia and its modifications) when NG showed low efficiency due to the rapid obliteration of the newly established ways of outflow of intraocular fluid. And only the active introduction of silicone drains and valves (Ahmed, Molteno, etc.) has opened a new chapter in surgical treatment of secondary NG at patients with DM. The low percentage of postoperative complications, ease of implantation and, most importantly, persistent postoperative hypotensive effect made them attractive for widespread use in this complex eye diseases, poorly amenable to various other methods of treatment.
The cases of "medium" proliferation while DR are rare [2]. For the entire period of observation we bumped into him only 2 times that is just $0,007 \%$. The growth of newly formed vessels on the AGM of the vitreous body, is possible only under condition of its pathological changes (seal). In the first case, the cause of fibrous transformation became old, almost total hemophthalmus, in the second, marked dislocation of the vitreous body forward towards the lens, a complete detachment of the PHM, and histological seal the remnants of the vitreous mass. Obviously, such a dense substrate as a modified anterior hyaloid membrane can be regarded as a favorable material for the development of medium proliferation. It should be noted that the initial patient symptoms front and rear of cell proliferation was detected, and another was pronounced robes of the iris and anterior chamber angle of the eye, and the patient was operated on the decompensated neovascular glaucoma with implantation of Ahmed valve.

The average proliferation in proliferative $D R$ can be combined with other options pathological neovascular process or can be an independent symptom. This does not preclude its classification as a severe lesion of the eyeball in patients with DM and requires a joint effort in the treatment of endocrinologists and ophthalmologists. Adequate and timely correction of the diabetic status, together with a vitreoretinal surgery can significantly improve the prognosis in this challenging group of patients and significantly reduce the percentage of disability. The holding of LCS in this pathology is not possible, and local use of the inhibitors of angiogenesis remains questionable.

\section{Conclusion}

Thus, proliferation in DR patients with diabetes can be observed in all departments of the eyeball. With the growth of abnormal blood vessels in the eye fundus by PGM vitreous body we're talking about "rear", histologically modified AGM - medium, in the corner of the anterior chamber of the eye and/or iris - "anterior" localization of the pathological proliferative process. This suggests that the formation of new functionally defective blood vessels is not a local, but generalized in nature and the impact on him must be the same complex. Timely laser coagulation of the retina, intravitreal injection of anti-VEGF, vitrectomy, drainage of surgery and other modern methods of treatment gives positive results and allows to look optimistically into the future.

Conflicts of Interest: The authors declare no conflicts of interest with this submission.

\section{References}

1. Nam Han Cho, David Whiting, Nita Forouhi, et al. IDF Diabetes Atlas. 7th edition. 2015.

2. Lipatov D.V, Tolkacheva A.A, Chistyakov T.A, et al. Features of proliferative process in patients with diabetic retinopathy-Diabetes Mellitus. 2th edition. 2012: 99-102.

3. Lipatov D.V. Diabetic glaucoma. Moscow, MIA; 2013: 183.

4. Lipatov D.V, Bessmertnaya E.G, Kuzmin A.G. et al. Epidemiology and register of diabetic retinopathy in the Russian Federation. Diabetes Mellitus. 2014; 1: 4-7. 